\title{
TRPML1 Participates in the Progression of Alzheimer's Disease by Regulating the PPARY/AMPK/Mtor Signalling Pathway
}

\author{
Lu Zhang $^{\mathrm{a}}$ Yu Fang ${ }^{\mathrm{b}} \quad$ Xuan Cheng $^{\mathrm{a}} \quad$ Yajun Lian $^{\mathrm{a}} \quad$ Hongliang Xu $^{\mathrm{a}}$ Zhaoshu Zeng ${ }^{\mathrm{c}}$ \\ Hongcan Zhu \\ aDepartment of Neurology, The First Affiliated Hospital of Zhengzhou University, Zhengzhou \\ bDepartment of Intensive Care Unit, The First Affiliated Hospital of Zhengzhou University, Zhengzhou, \\ 'Department of Legal Medicine, The College of Basic Medical Sciences of Zhengzhou University, \\ Zhengzhou, China
}

\section{Key Words}

Trpml1 • PPAR $/$ AMPK/ mTOR/S6K signalling pathway • Alzheimer's disease

\begin{abstract}
Background: TRPML1 is reported to be involved in the pathogenesis of Alzheimer's disease (AD) by regulating autophagy; however, the underlying mechanism is not completely clear. Methods: We developed an APP/PS1 transgenic animal model that presents with AD. TRPML1 was also overexpressed in these mice. Protein expression levels were determined by Western blot. Morris water maze (MWM) and recognition tasks were performed to characterize cognitive ability. TUNEL assays were analysed for the detection of neuronal apoptosis. Primary neurons were isolated and treated with the vehicle, $A \beta 1-42$ or $A \beta 1-42+$ mTOR activator, as well as infected with the recombinant adenovirus TRPML1 overexpression vector in vitro. Cell viability was measured by the MTS assay, and lysosomal $\mathrm{Ca}^{2+}$ was also measured. Results: In the APP/PS1 transgenic mice, TRPML1 was downregulated, the PPAR $\gamma / A M P K$ signalling pathway was activated, the mTOR/S6K signalling pathway was suppressed, and autophagic lysosome reformation (ALR)-related proteins were upregulated. TRPML1 overexpression or treatment with PPARY and AMPK inhibitors or an mTOR activator reduced the expression levels of ALRrelated proteins, rescued the memory and recognition impairments and attenuated neuronal apoptosis in mice with the APP/PS1 transgenes. In vitro experiments showed that TRPML1 overexpression or treatment with the mTOR activator propranolol attenuated the A $31-42-$ suppressed cell viability and the A $A 1-42$-decreased lysosomal $\left[\mathrm{Ca}^{2+}\right]$ ion concentration in primary neurons. TRPML1 overexpression or treatment with the mTOR activator propranolol also attenuated the A $\beta 1-42$-inhibited mTOR/S6K signalling pathway and the A $\beta 1-42$-induced ALR-related protein expression levels. Conclusion: TRPML1 is involved in the pathogenesis of $A D$ by regulating autophagy at least in part through the PPARY/AMPK/mTOR signalling pathway.




\section{Cellular Physiology Cell Physiol Biochem 2017;43:2446-2456

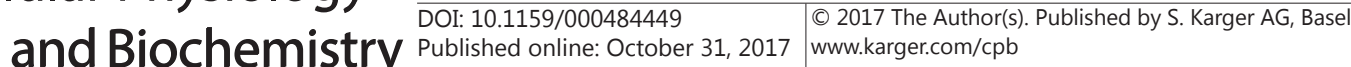

Zhang et al.: TRPML1 is Involved in the Pathogenesis of AD

\section{Introduction}

Alzheimer's disease (AD) is the most common neurodegenerative disorder in the world and accounts for more than $60 \%$ of all cases of dementia. AD is characterized by severe cognitive impairments and progressive memory loss that affect the life and health of elderly people $[1,2]$. The pathological characteristics of AD include the loss of pyramidal neurons, extracellular accumulation of senile plaques that contain amyloid- $\beta(A \beta)$ deposits, and neurofibrillary tangles that contain hyper-phosphorylated tau [3, 4]. Autophagy is a highly conserved intracellular pathway involved in the organized elimination of proteins and organelles by lysosomes, and it is both required for cellular adaptation to nutrient deprivation and stress and important for neuronal survival [5]. Abnormalities in the lysosomal system have been identified in neurons of the AD brain, including a massive buildup of incompletely digested substrates within both dystrophic axons and dendrites and pathological endocytic pathway responses at the very earliest disease stage [6]. Moreover, autophagy not only exerts a degradative function but also mediates the secretion of $A \beta$, indicating a vital role for autophagy in the homeostasis of $A \beta$ and placing it in a very unique and intriguing position in AD pathogenesis [7]. In particular, during autophagy, lysosomes fuse with autophagosomes to form autolysosomes. Following starvation-induced autophagy, nascent lysosomes are formed from autolysosomal membranes through an evolutionarily conserved cellular process called autophagic lysosome reformation (ALR) that is critical for maintaining lysosome homeostasis [8]. Since autophagy is strongly associated with AD pathogenesis and ALR is critical for maintaining lysosome homeostasis, it seems that ALR also participates in $\mathrm{AD}$ progression.

Transient receptor potential Mucolipin-1 (TRPML1) is widely expressed in mammalian cell lysosomes or in the endosome membrane [9] and is the main channel for lysosome $\mathrm{Ca}^{2+}$ release and the key regulator for lysosomal storage and transportation [10]. In addition, TRPML1 also plays an important role in signal transduction and maintenance of lysosome acid homeostasis [11]. TRPML1 mutations can not only induce the occurrence of neurodegenerative lysosomal storage disorders but also affect the accumulation of autophagy. Thus, TRPML1 is considered to be the regulator of autophagy $[9,12]$. Importantly, a recent study suggested that, in a triple transgenic gp120/APP/PS1 mouse, the HIV coat protein gp120 inhibits the activity of TRPML1 and thereby facilitates the intraneuronal accumulation of $A \beta[13]$. This finding raises the possibility that TRPML1 is involved in the pathogenesis of $\mathrm{AD}$ by regulating autophagy; however, the underlying mechanism is not clear completely.

Considering that the activation of peroxisome proliferator-activated receptor $\gamma$ (PPAR $\gamma$ ) has been reported to induce autophagy and that the AMP activated protein kinase/ mammalian target of rapamycin/p70ribosomal S6 Kinase (AMPK/mTOR/S6K) is the predominant signalling pathway that regulates autophagy [14], we speculated that TRPML1 is involved in the pathogenesis of $\mathrm{AD}$ by regulating autophagy via the PPAR $\gamma / \mathrm{AMPK} / \mathrm{mTOR}$ signalling pathway. Our study was designed to test this hypothesis.

\section{Materials and Methods}

\section{Animals}

APP/PS1 transgenic mice were purchased from the animal model centre of Nanjing University (Nanjing, China) and were maintained on a C57BL/6J background by crossing heterozygous transgenic mice with C57BL/6J breeders. Mice with TRPML1 overexpression were constructed as previously described [15]. The remaining mice were divided into 3 groups: one group received an intravenous injection of AMP inhibitor 8-bromo-AMP (8-bAMP) ( $3 \mathrm{mg} / \mathrm{kg}$; Sta. Cruz Biotechnology, Santa Cruz, CA), one group received an intraperitoneal injection of PPAR $\gamma$ antagonist GW9662 (1 mg/kg, St Louis, MO, U.S.A.), and the other group received a dose of the mTOR-activator propranolol. This study was carried out in strict accordance with the recommendations of the Guide for the Care and Use of Laboratory Animals of the National Institutes of Health. The protocol was approved by the Ethics Committee of the Animal Experiments of the University of Zhengzhou. 


\section{Cellular Physiology Cell Physiol Biochem 2017;43:2446-2456 \begin{tabular}{l|l} 
and Biochemistry Published online: October 31, 2017 & $\begin{array}{l}\text { C } 2017 \text { The Author(s). Published by S. Karger AG, Basel } \\
\text { www.karger.com/cpb }\end{array}$ \\
\hline
\end{tabular}}

Zhang et al.: TRPML1 is Involved in the Pathogenesis of AD

A 1-42 preparation and the isolation of primary neurons and cell culture

Aß1-42 preparation was performed as previously described [16]. Mixed cortical and hippocampal neurons were isolated from embryos on embryonic day 14-16, as previously described [17]. Cells were maintained in Dulbecco's modified Eagle's medium (D-MEM; Nacalai tesque, Kyoto, Japan) containing 10\% foetal bovine serum, 100 units/mL penicillin, and $100 \mu \mathrm{g} / \mathrm{mL}$ streptomycin in a humidified atmosphere of $95 \%$ air and $5 \% \mathrm{CO}_{2}$ at $37^{\circ} \mathrm{C}$. Primary neuronal cultures were then treated with vehicle, $5 \mathrm{uM} \mathrm{A} \beta 1-42$, or $5 \mathrm{uM}$ $A \beta 1-42+$ propranolol for up to 72 hours in treatment. Recombinant adenovirus TRPML1 overexpression vector was constructed and infected into neurons as previously described [18].

MTS assay

Cell viability was measured by the cellular ability to metabolize MTS (Promega Corporation, Madison, USA) in the presence of phenazine methosulfate to a formazan product, as described previously [17]. The results were expressed as a percentage of the absorbance at $490 \mathrm{~nm}$ of the control vehicle.

The Morris water maze (MWM) test

The MWM test was performed to evaluate the escape latency (the time to reach the hidden platform), travelled distance (the length of swim path) and time across the platform, as described in previous studies $[19,20]$. Recognition tasks were performed as described previously [21].

\section{Calcium measurements}

Lysosomal $\mathrm{Ca}^{2+}$ was measured using low-affinity rhodamine B dextran (10 kDa) in conjunction with $\mathrm{Ca}^{2+}$-insensitive Alexa Fluor 488 dextran $(0.25 \mathrm{mg} / \mathrm{mL}$; Invitrogen, Carlsbad, CA, USA), as described previously [22].

\section{Western blot analysis}

Sample proteins were extracted and separated by SDS-polyacrylamide gel electrophoresis and transferred to a polyvinylidene difluoride membrane (Millipore, Germany). The blots were incubated with the primary antibodies anti-TRPML1, anti- PPAR $\gamma$, anti-S6K, anti-p-S6K, anti-AMPK, anti-p-AMPK, antimTOR, anti-p-mTOR, anti-Beclin, anti-LC3, anti-LAMP- 1 and anti- $\beta$-actin overnight at $4{ }^{\circ} \mathrm{C}$. The membranes were washed and then incubated with horseradish peroxidase-conjugated secondary antibodies (SigmaAldrich, St Louis, MO, USA) for 1 hour. The immunoreactive proteins were visualized with SuperSignal ECL (Pierce; Rockford, IL, USA), and the densities of the bands were analysed by ImageJ.

\section{Statistical analyses}

Data were presented as the mean \pm standard error of the mean and analysed by using SPSS18.0. Comparisons among multiple groups were examined by 1- or 2-way analysis of variance followed by Fischer's protected least significant difference post hoc tests. Comparisons between two groups were analysed by Student's 2-tailed unpaired $t$-test. Significant differences were indicated by a $p$ value $<0.05$.

\section{Results}

TRPML1 was downregulated, the PPAR $/ A M P K$ signalling pathway was activated, the mTOR/S6K signalling pathway was inhibited, and the ALR-related proteins were upregulated in APP/PS1 transgenic mice

The hippocampus was collected and used for Western blotting analysis. The results showed that the protein expression level of TRPML1 was downregulated in APP/PS1 transgenic mice compared with the control animals. Importantly, the protein expression level of PPAR $\gamma$ was upregulated in APP/PS1 transgenic mice compared with that in the controls (Fig. 1A). The presence of the APP/PS1 transgenes in the mice also decreased the protein expression levels of $\mathrm{p}-\mathrm{S} 6 \mathrm{~K}$ and $\mathrm{p}-\mathrm{mTOR}$ and increased the expression of $\mathrm{p}-\mathrm{AMPK}$ compared to that in the controls, indicating activation of the PPAR $\gamma /$ AMPK signalling pathway and inhibition of the mTOR/S6K signalling pathway (Fig. 1B). Beclin, LC3 and LAMP-1 are markers of the autophagy-lysosomal system [23]. The protein expression levels of Beclin, LC3 and LAMP-1 were upregulated in mice with APP/PS1 transgenes compared with levels KARGER 


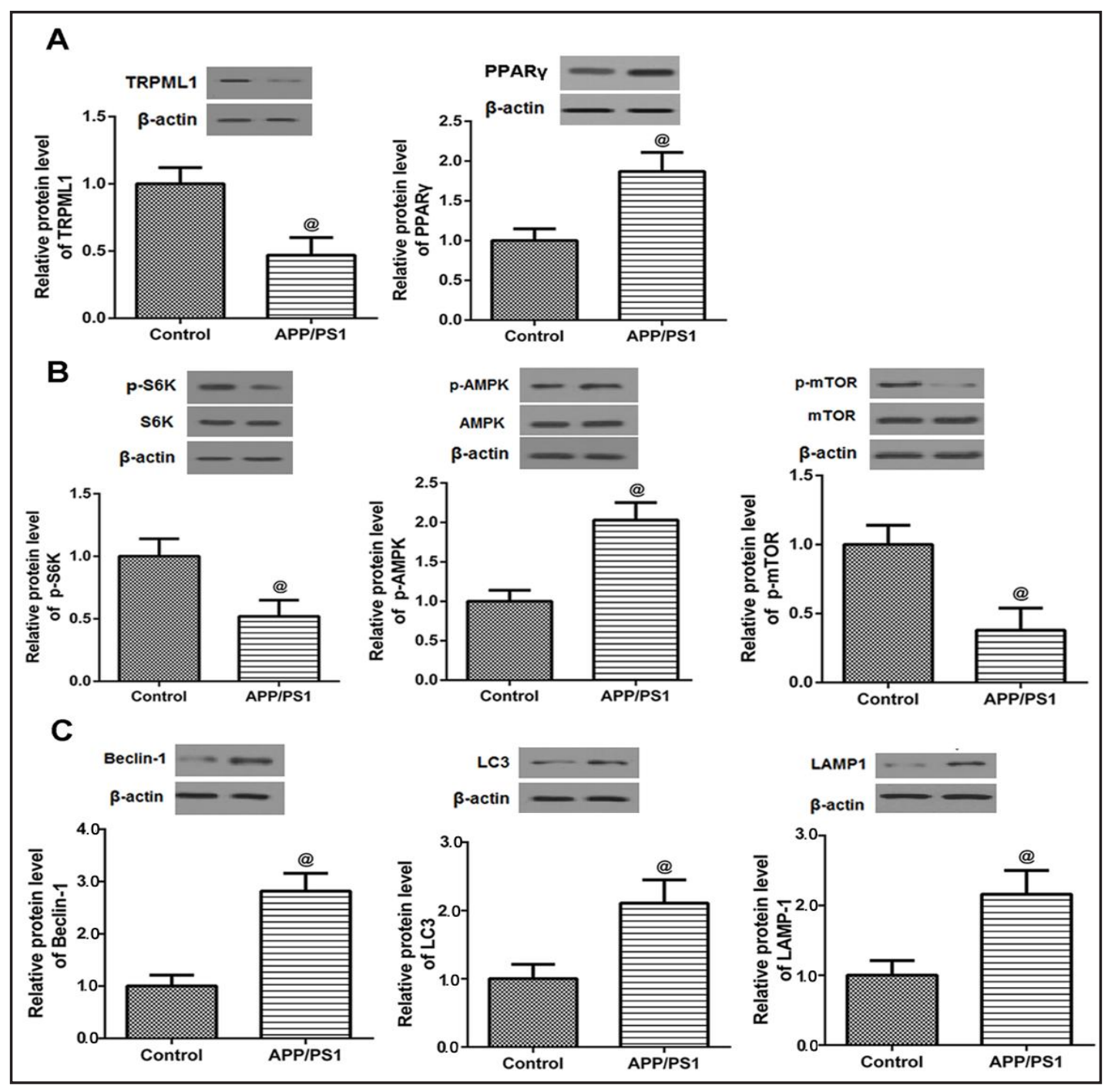

Fig. 1. TRPML1 was downregulated, the PPAR $\gamma / A M P K$ signalling pathway was activated, the mTOR/S6K signalling pathway was inhibited, and the ALR-related proteins were upregulated in APP/PS1 transgenic mice(A) The protein expression level of TRPML1 was downregulated in the APP/PS1 transgenic mice compared with that in the controls ( $\mathrm{p}<0.05 ; \mathrm{n}=8$ in each group). (B) The presence of the APP/PS1 transgenes in mice decreased the protein expression levels of p-S6K and p-mTOR but increased the protein expression level of $p$-AMPK compared to that in the controls ( $p<0.05 ; n=8$ in each group). (C) The protein expression levels of Beclin, LC3 and LAMP-1 were upregulated in mice with the APP/PS1 transgenes in comparison with the levels in the controls ( $\mathrm{p}<0.05 ; \mathrm{n}=8$ in each group). @ $\mathrm{p}<0.05$ compared to controls.

in the controls (Fig. 1C). These results indicate the potential role of TRPML1 and the PPAR $\gamma /$ AMPK/ mTOR signalling pathway in AD progression.

TRPML1 overexpression, PPAR $\gamma$ and AMPK inhibitors, and an mTOR activator reduced the expression levels of ALR-related proteins in APP/PS1 transgenic mice

To validate the potential role of TRPML1 and the PPAR $\gamma /$ AMPK/mTOR signalling pathway in AD progression, TRPML1 was overexpressed in mice with the APP/PS1 transgenes, or the transgenic mice were treated with the AMP inhibitor 8-bAMP, the PPAR $\gamma$ antagonist GW9662 or the mTOR activator propranolol. We found that TRPML1 was significantly upregulated in the APP/PS1 model with TRPML1 overexpression compared with the APP/PS1 mice (Fig. 2A). We also observed that TRPML1 overexpression significantly increased the protein 
Fig. 2. TRPML1 overexpression and the inhibitors of the PPAR $\gamma /$ AMPK signalling pathway reduced the expression levels of ALR-related proteins in the APP/PS1 transgenic mice(A) TRPML1 was significantly upregulated in the APP/PS1 model with TRPML1 overexpression compared with the APP/ PS1 mice $(p<0.05 ; n=8$ in each group). (B) TRPML1 overexpression, the PPAR $\gamma$ and AMPK inhibitors and the mTOR activator induced the protein expression levels of $\mathrm{p}-\mathrm{S} 6 \mathrm{~K}$ and p-mTOR in mice with APP/ PS1 transgenes compared with mice without treatment $(\mathrm{p}<0.05 ; \mathrm{n}=8$ in each group). (C) TRPML1 overexpression, the PPAR $\gamma$ and AMPK inhibitors and the mTOR activator reduced the protein expression levels of Beclin, LC3 and LAMP-1 in mice with the APP/PS1 transgenes compared with mice without treatment $(\mathrm{p}<0.05 ; \mathrm{n}=8$ in each group). @p $<0.05$ compared to controls; $\# \mathrm{p}<0.05$ compared to the APP/PS1 group.
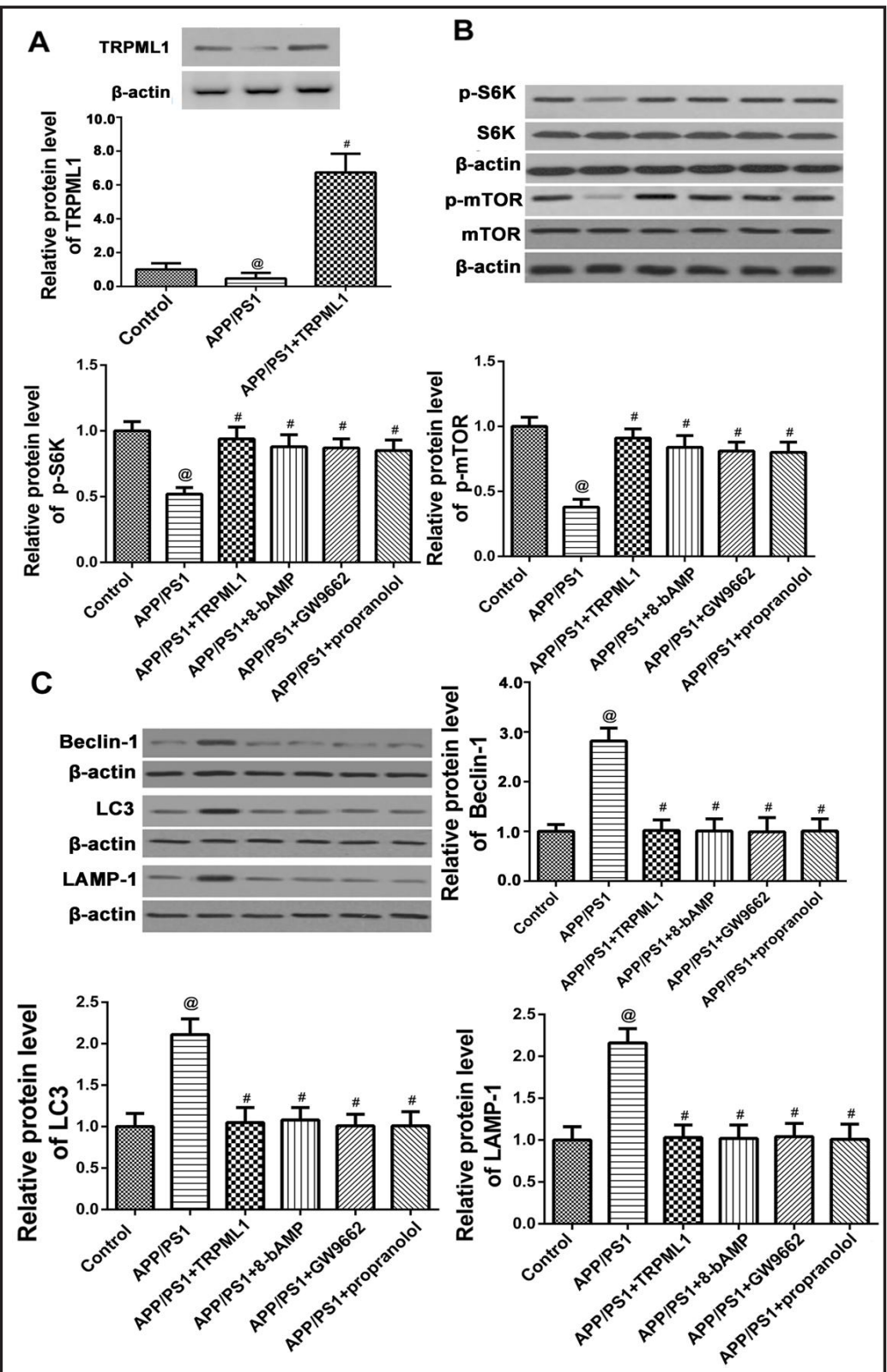

expression levels of $\mathrm{p}$-S6K and p-mTOR in mice with the APP/PS1 transgenes in comparison with the APP/PS1 mice, which is comparable to the effects of the AMP inhibitor 8-bAMP, the PPAR $\gamma$ antagonist GW9662 and the mTOR activator propranolol (Fig. 2B). We also found that TRPML1 overexpression significantly decreased the protein expression levels of Beclin, LC3 and LAMP-1 in mice with the APP/PS1 transgenes compared with the APP/PS1 mice. In addition, after treatment with the AMP inhibitor 8-bAMP, the PPAR $\gamma$ antagonist GW9662 or the mTOR activator propranolol in mice with the APP/PS1 transgenes, the protein expression levels of the ALR-related proteins Beclin, LC3 and LAMP-1 were also reduced (Fig. 2C).

TRPML1 overexpression, PPAR $\gamma$ and AMPK inhibitors, and an mTOR activator rescued the memory and recognition impairments and neuronal apoptosis in mice with the APP/PS1 transgenes

We then found that the presence of APP/PS1 transgenes caused significant inductions in escape latency (Fig. 3A), travel length (Fig. 3B) and time across the platform (Fig. 3C) in 
A

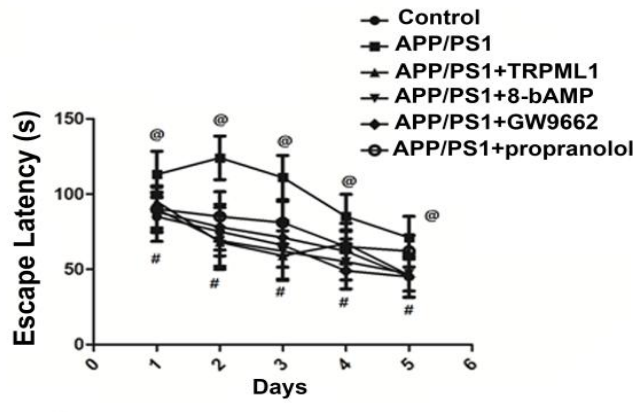

C

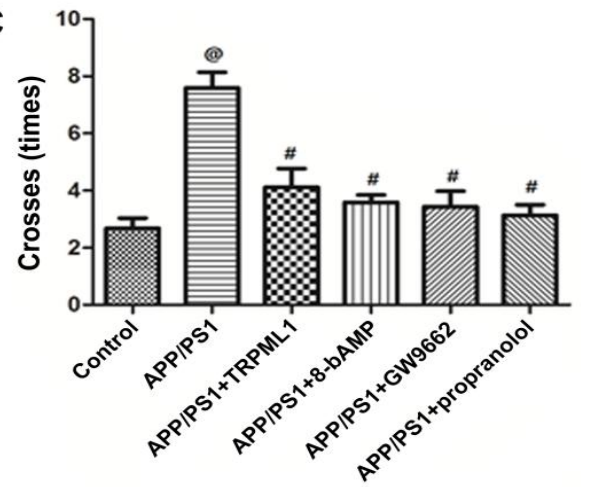

E
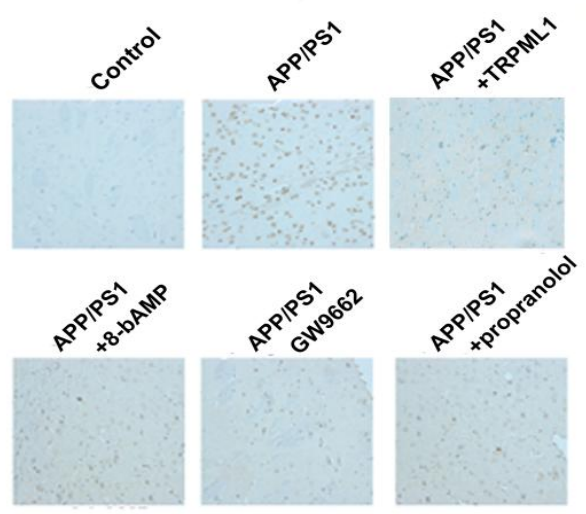

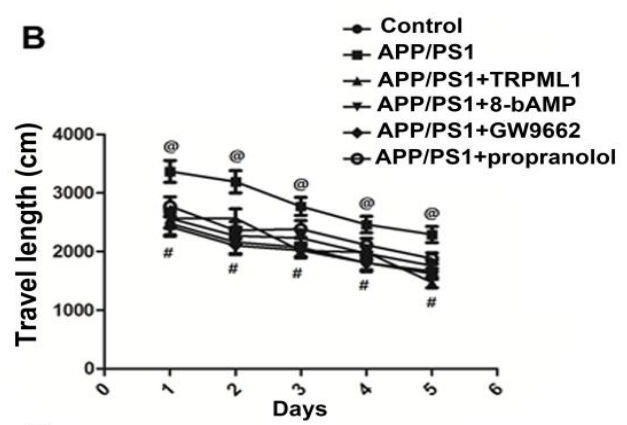

D
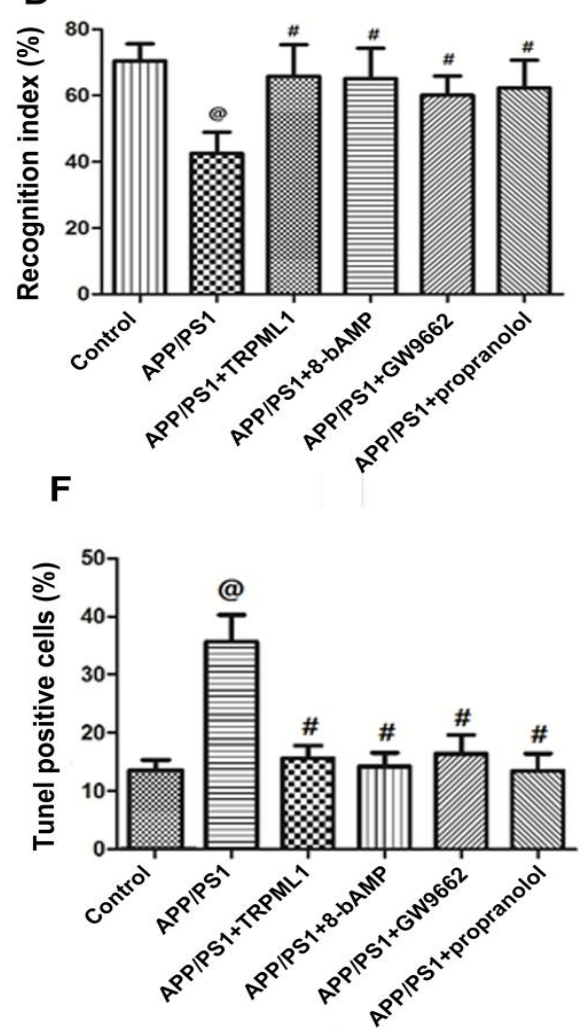

Fig. 3. TRPML1 overexpression and the inhibitors of the PPAR $\gamma / A M P K / m T O R$ signalling pathway rescued the memory and recognition impairments and neuronal apoptosis in mice with the APP/PS1 transgenesTRPML1 overexpression or treatment with 8-bAMP, GW9662, or propranolol caused significant reductions in the escape latency (A), travel length (B), and time across the platform (C) and markedly improved the recognition index (D) in the APP/PS1 transgenic mice compared to mice without treatment $(\mathrm{p}<0.05$; $n=8$ in each group). (E-F) The neuronal apoptotic rate in the APP/PS1 transgenic mice with TRPML1 overexpression or treatment with 8-bAMP, GW9662 or propranolol was markedly reduced compared to that in mice without treatment ( $\mathrm{p}<0.05 ; \mathrm{n}=8$ in each group). @ $\mathrm{p}<0.05$ compared to controls; $\# \mathrm{p}<0.05$ compared to the APP/PS1 group.

comparison with the results for the controls. The presence of APP/PS1 transgenes caused a significant reduction in the recognition index compared with that in the controls (Fig. 3D). TRPML1 overexpression or treatment with 8-bAMP, GW9662, or propranolol caused significant reductions in escape latency (Fig. 3A), travel length (Fig. 3B), and time across the platform (Fig. 3C) and markedly improved the recognition index (Fig. 3D) in APP/ PS1 transgenic mice compared to mice without treatment. TUNEL staining was used to detect neuronal apoptosis in AD mice. As shown in Fig. 3E and 3F, the presence of APP/ 
A

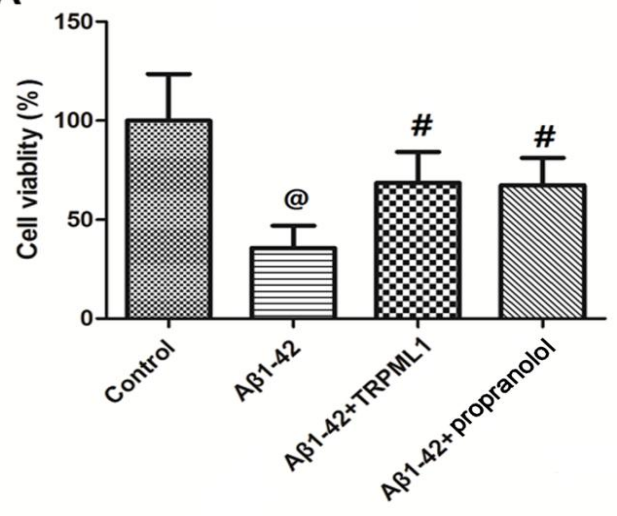

B

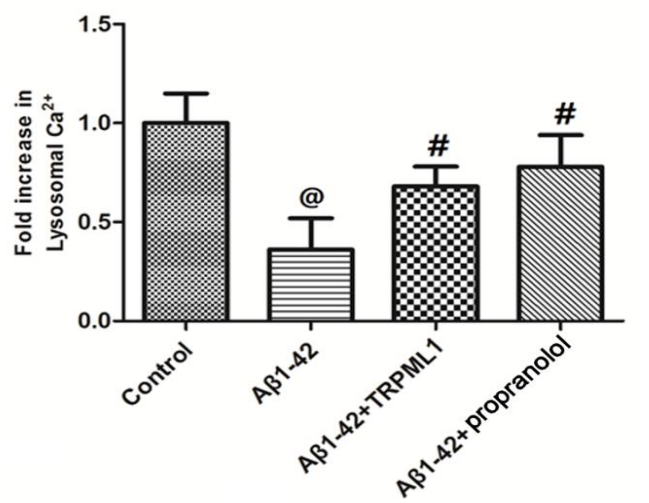

Fig. 4. TRPML1 overexpression or the mTOR activator propranolol attenuated the Aß1-42-suppressed cell viability and the Aß1-42-decreased lysosomal $\left[\mathrm{Ca}^{2+}\right]$ ion concentration in vitro(A) TRPML1 overexpression or the mTOR activator propranolol markedly attenuated the Aß1-42-suppressed cell viability in neurons in vitro $(\mathrm{p}<0.05)$. (B) TRPML1 overexpression or the mTOR activator propranolol significantly attenuated the $A \beta 1-42$-decreased lysosomal $\left[\mathrm{Ca}^{2+}\right]$ ion concentration in vitro $(\mathrm{p}<0.05)$. @ $\mathrm{p}<0.05$ compared to controls; $\# \mathrm{p}<0.05$ compared to the $A \beta 1-42$-treated group.

PS1 transgenes caused a significant increase in the neuronal apoptotic rate compared with that in the controls. Importantly, a marked reduction in the neuronal apoptotic rate in the APP/PS1 transgenic mice was found with TRPML1 overexpression or with 8-bAMP, GW9662 or propranolol treatment. These investigations suggested that TRPML1 overexpression or inhibitors of the PPAR $\gamma /$ AMPK signalling pathway prevented the dysfunctions of spatial memory and recognition, as well as neuronal apoptosis.

TRPML1 overexpression or the mTOR activator propranolol attenuated the A $\beta 1-42$ suppressed cell viability and the Aß1-42-decreased lysosomal $\left[\mathrm{Ca}^{2+}\right]$ ion concentration in vitro

Next, primary neurons were isolated from the hippocampus and treated with A $\beta 1-42$ in vitro. Our results showed that cell viability was significantly inhibited in A $\beta 1$-42-treated neurons compared with the controls. In contrast, TRPML1 overexpression or treatment with the mTOR activator propranolol markedly attenuated the AB1-42-suppressed cell viability of neurons in vitro (Fig. 4A). Furthermore, A 1 1-42 treatment caused a significant decrease in the lysosomal $\left[\mathrm{Ca}^{2+}\right]$ ion concentration compared with the control, and TRPML1 overexpression or treatment with the mTOR activator propranolol significantly attenuated the Aß1-42-mediated decrease in lysosomal $\left[\mathrm{Ca}^{2+}\right]$ ion concentration (Fig. 4B).

TRPML1 overexpression or the mTOR activator propranolol attenuated the Aß1-42inhibited mTOR/S6K signalling pathway and the AB1-42-induced ALR-related protein expression levels in vitro

As shown in Fig. 5, our results showed that the protein expression levels of p-S6K and p-mTOR were inhibited in neurons with A 31 -42-treatment compared with the controls. Inversely, TRPML1 overexpression or the MTOR activator propranolol markedly attenuated the A 31 -42-inhibited mTOR/S6K signalling pathway, inducing the protein expression of p-S6K and p-mTOR. In addition, A $\beta 1-42$ treatment caused increased expression levels of the ALR-related proteins Beclin, LC3 and LAMP-1 compared with the controls, and TRPML1 overexpression or the mTOR activator propranolol significantly attenuated the A $\beta 1-42$ induced ALR-related protein expression levels. 


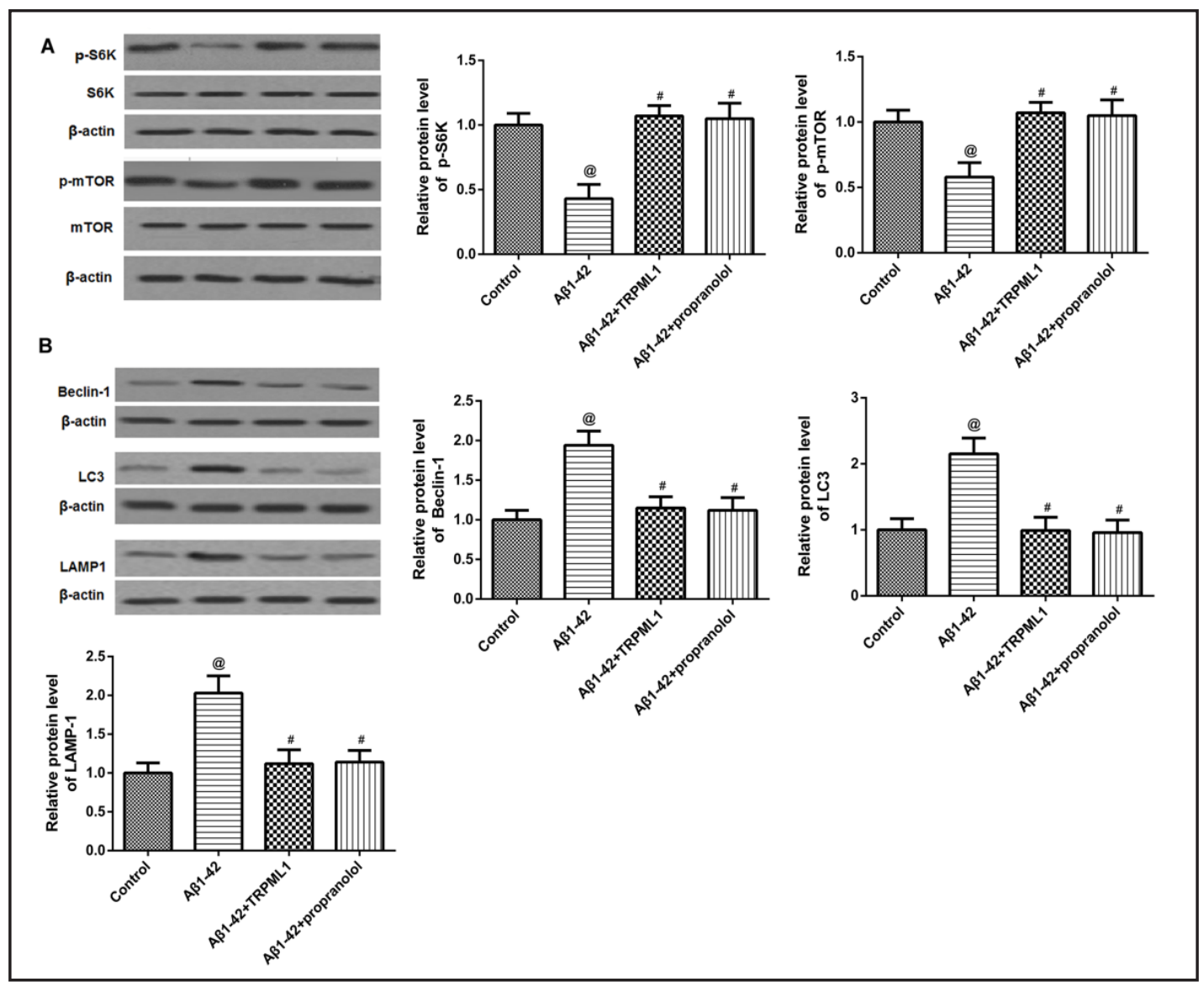

Fig. 5. TRPML1 overexpression or the mTOR activator propranolol attenuated the A $\beta 1$-42-inhibited mTOR/ S6K signalling pathway and the A $\beta 1$-42-induced ALR-related protein expression levels in vitro (A) TRPML1 overexpression or the mTOR activator propranolol markedly attenuated the A $\beta 1$-42-inhibited mTOR/S6K signalling pathway in vitro $(\mathrm{p}<0.05)$. (B) TRPML1 overexpression or the mTOR activator propranolol significantly attenuated the A $\beta 1$-42-induced ALR-related protein expression levels in vitro $(\mathrm{p}<0.05)$. @p $<0.05$ compared to controls; \#p<0.05 compared to the A $\beta 1$-42-treated group.

\section{Discussion}

The mucolipin subfamily of TRP proteins (TRPMLs) contain six transmembranespanning proteins that consist of cytosolic $\mathrm{N}$ - and C-termini. There are three TRPML proteins, TRPML1 (also called MCOLN1), TRPML 2 (also called MCOLN2) and TRPML 3 (also called MCOLN3), in mammals [9]. Loss-of-function mutations in the human TRPML1 gene cause type IV mucolipidosis (ML4), a devastating neurodegenerative disease that causes retinal degeneration and mental retardation [9, 24]. Additionally, TRPML1-/cells from ML4 patients are characterized by the accumulation of enlarged endosomal/ lysosomal compartments (vacuoles) in which lipids and other biomaterials accumulate, suggesting defective lysosomal biogenesis and trafficking $[9,25]$. In addition, a previous study suggested that a TRPML1 agonist facilitates the lysosomal clearance of proteins and lipids in a mouse model of gp120-facilitated amyloid deposition [26]. To some extent, these investigations showed the potential role of TRPML1 in AD progression. Indeed, we found that TRPML1 was downregulated in APP/PS1 transgenic mice, and TRPML1 overexpression reduced the expression levels of ALR-related proteins, rescued both memory and recognition impairments and attenuated neuronal apoptosis in mice with APP/PS1 transgenes in vivo. In addition, TRPML1 overexpression attenuated A $\beta 1$-42-suppressed cell viability, $A \beta 1-42$ -

\section{KARGER}


decreased lysosomal $\left[\mathrm{Ca}^{2+}\right]$ ion concentration, and A $\beta 1$-42-induced ALR-related protein expression levels in vitro.

A previous study indicated that the PPAR $\gamma$ agonist troglitazone induces autophagy, apoptosis and necroptosis in bladder cancer cells [27]. However, rosiglitazone, a selective PPAR- $\gamma$-inhibitor, is reported to induce autophagy in H295R cells and cell cycle deregulation in SW13 adrenocortical cancer cells [28]. It seems that the role of PPAR $\gamma$ in autophagy remains unclear. PPAR $\gamma$ agonists are considered new therapeutic agents for the treatment of AD[29]. These protective effects are primarily considered to result from the anti-inflammatory and anti-oxidant actions of PPAR $\gamma[30]$. Moreover, other studies showed that autophagy is not only affected by AMPK, a key energy sensor that regulates cellular metabolism to maintain energy homeostasis, but also affected by mTOR, a central cell-growth regulator that integrates growth factors and nutrient signals [31, 32]. Autophagy is promoted by AMPK, and the mTOR/S6K signalling pathway is well known to negatively regulate autophagy [31, 33]. A previous study indicated that increased mTOR activity attenuates autophagy and generates proto-lysosomal tubules and vesicles, which extrude from autolysosomes and ultimately mature into functional lysosomes that restore the full complement of lysosomes to the cell, revealing its vital role in ALR [34]. Moreover, Li et al. revealed that the induction of autophagy by telmisartan occurred with a dose-dependent increase in the expression of PPAR $\gamma$ and p-AMPK and a decrease in the expression of p-mTOR, whereas GW9662 attenuated these expression levels [14]. Similarly, we found that the presence of the APP/PS1 transgenes in mice increased the protein expression level of p-AMPK but decreased the expression levels of p-S6K and p-mTOR. The AMP inhibitor 8-bAMP, the PPAR $\gamma$ antagonist GW9662 and the mTOR activator propranolol reduced the expression levels of ALR-related proteins, rescued the memory and recognition impairments and attenuated neuronal apoptosis in mice with the APP/PS1 transgenes in vivo. In addition, propranolol attenuated A $\beta 1-42$-suppressed cell viability, A $\beta 1-42$-decreased lysosomal $\left[\mathrm{Ca}^{2+}\right]$ ion concentration, and A $\beta 1-42$-induced ALRrelated protein expression levels in vitro.

Importantly, TRPML1 overexpression reduced the protein expression level of p-AMPK and induced the protein expression levels of p-S6K and p-mTOR in mice with the APP/ PS1 transgenes in comparison with the controls. TRPML1 overexpression also induced protein expression levels of p-S6K and p-mTOR in the A $31-42$-treated cells compared with the controls. The effects of 8-bAMP, GW9662 or propranolol on rescuing the memory and recognition impairments and attenuating neuronal apoptosis, as well as the effects of propranolol on reversing $A \beta 1-42$-suppressed cell viability, $A \beta 1-42$-decreased lysosomal $\left[\mathrm{Ca}^{2+}\right]$ ion concentration and A $\beta 1-42$-induced ALR-related protein expression levels, were comparable to the effects of TRPML1 overexpression. Thus, it seems that TRPML1 is involved in the pathogenesis of $\mathrm{AD}$ at least partially through the regulation of autophagy via the PPAR $\gamma / \mathrm{AMPK} / \mathrm{mTOR}$ signalling pathway.

\section{Acknowledgements}

This research was in part supported by grants from the Zhengzhou Science \& Technology Basic Research Program (No. 131PPTGG409-21).

\section{Disclosure Statement}

The authors declare that there are no conflicts of interest.

\section{References}

1 Yin R, Yin K, Guo Z, Zhang Z, Chen L, Cao L, Li Y, Wei Y, Fu X, Shi X: Protective Effects of Colivelin Against Alzheimer's Disease in a PDAPP Mouse Model. Cell Physiol Biochem 2016;38:1138.

\section{KARGER}




\section{Cellular Physiology Cell Physiol Biochem 2017;43:2446-2456

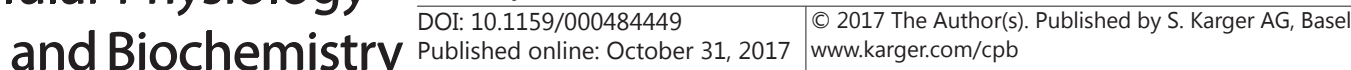 \\ Zhang et al.: TRPML1 is Involved in the Pathogenesis of AD}

-2 Liu Z, Wang C, Wang X, Xu S: Therapeutic Effects of Transplantation of As-MiR-937-Expressing Mesenchymal Stem Cells in Murine Model of Alzheimer's Disease. Cell Physiol Biochem 2015;37:321-330.

-3 Selkoe DJ: Alzheimer's disease results from the cerebral accumulation and cytotoxicity of amyloid betaprotein. J Alzheimers Dis 2001;3: 75-80

-4 Grundke-Iqbal I, Iqbal K, Tung YC, Quinlan M, Wisniewski HM, Binder LI: Abnormal phosphorylation of the microtubule-associated protein tau (tau) in Alzheimer cytoskeletal pathology. Proc Natl Acad Sci U S A 1986;83:4913-4917.

-5 Tung YT, Wang BJ, Hu MK, Hsu WM, Lee H, Huang WP, Liao YF: Autophagy: A double-edged sword in Alzheimer's disease. J Biosci 2012;37:157-165.

-6 Nixon RA, Yang DS: Autophagy failure in Alzheimer's disease-locating the primary defect. Neurobiol Dis 2011;43:38-45.

7 Nilsson P, Saido TC: Dual roles for autophagy: Degradation and secretion of Alzheimer's disease A $\beta$ peptide. Bioessays 2014;36:570-578.

8 Rong Y, Liu M, Ma L, Du W, Zhang H, Tian Y, Cao Z, Li Y, Ren H, Zhang C: Clathrin and phosphatidylinositol-4, 5-bisphosphate regulate autophagic lysosome reformation. Nat Cell Biol 2012;14:924-934.

-9 Cheng X, Shen D, Samie M, Xu H: Mucolipins: Intracellular TRPML1-3 channels. FEBS Lett 2010;584:20132021.

10 Li X, Garrity AG, Xu H: Regulation of membrane trafficking by signalling on endosomal and lysosomal membranes. J Physiol 2013;591:4389-4401.

11 Morgan AJ, Platt FM, Lloydevans E, Galione A: Molecular mechanisms of endolysosomal Ca2+ signalling in health and disease. Biochem J 2011;439:349-374.

-12 Curcio-Morelli C, Charles FA, Micsenyi MC, Cao Y, Venugopal B, Browning MF, Dobrenis K, Cotman SL, Walkley SU, Slaugenhaupt SA: Macroautophagy is defective in mucolipin-1-deficient mouse neurons. Neurobiol Dis 2010;40:370-377.

13 Bae M, Patel N, Xu H, Lee M, Tominaga-Yamanaka K, Nath A, Geiger JD, Gorospe MM, Mattson MP, Haughey NJ: Activation of TRPML1 Clears Intraneuronal A beta in Preclinical Models of HIV Infection. J Neurosci 2014;34:11485-11503.

-14 Li BH, Liao SQ Yin YW, Long CY, Guo L, Cao XJ, Liu Y, Zhou Y, Gao CY, Zhang LL: Telmisartan-induced PPAR activity attenuates lipid accumulation in VSMCs via induction of autophagy. Mol Biol Rep 2014;42:179-186.

15 Tóth ME, Szegedi V, Varga E, Juhász G, Horváth J, Borbély E, Csibrány B, Alföldi R, Lénárt N, Penke B: Overexpression of Hsp27 ameliorates symptoms of Alzheimer's disease in APP/PS1 mice. Cell Stress Chaperones 2013;18:759-771.

16 Troy CM, Rabacchi SA, Xu Z, Maroney AC, Connors TJ, Shelanski ML, Greene LA: beta-Amyloid-induced neuronal apoptosis requires c-Jun N-terminal kinase activation. J Neurochem 2001;77:157-164.

17 Taylor JM, Minter MR, Newman AG, Zhang M, Adlard PA, Crack PJ: Type-1 interferon signaling mediates neuro-inflammatory events in models of Alzheimer's disease. Neurobiol Aging 2014;35:1012-1023.

18 Tamatani M, Che YH, Matsuzaki H, Ogawa S, Okado H, Miyake S, Mizuno T, Tohyama M: Tumor Necrosis Factor Induces Bcl-2 and Bcl-x Expression through NFkB Activation in Primary Hippocampal Neurons. J Biol Chem 1999;274:8531-8538.

19 Morris R: Development of a water-maze procedure for studying spatial learning in the rat. J Neurosci Methods 1984;11:47-60.

20 Wang J, Yuan J, Pang J, Ma J, Han B, Geng Y, Shen L, Wang H, Ma Q, Wang Y: Effects of Chronic Stress on Cognition in Male SAMP8 Mice. Cell Physiol Biochem 2016;39:1078.

21 Kim DH, Ryu JH: Differential Effects of Scopolamine on Memory Processes in the Object Recognition Test and the Morris Water Maze Test in Mice. Biomol Ther2008;16:173-178.

22 Coen K, Flannagan RS, Baron S, Carrarolacroix LR, Wang D, Vermeire W, Michiels C, Munck S, Baert V, Sugita S: Lysosomal calcium homeostasis defects, not proton pump defects, cause endo-lysosomal dysfunction in PSEN-deficient cells. J Cell Biol 2012;198:23.

23 Castino R: Chelation of lysosomal iron protects dopaminergic SH-SY5Y neuroblastoma cells from hydrogen peroxide toxicity by precluding autophagy and Akt dephosphorylation. Toxicol Sci 2011;123:523-541.

24 Sun M, Goldin E, Stahl S, Falardeau JL, Kennedy JC, Jr AJ, Bove C, Kaneski CR, Nagle J, Bromley MC: Mucolipidosis type IV is caused by mutations in a gene encoding a novel transient receptor potential channel. Hum Mol Genet 2000;9:2471-2478. 


\section{Cellular Physiology Cell Physiol Biochem 2017;43:2446-2456

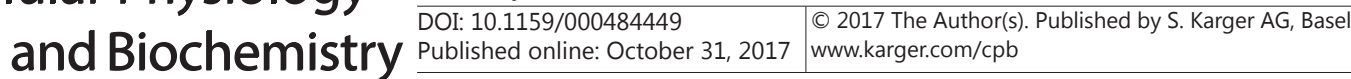 \\ Zhang et al.: TRPML1 is Involved in the Pathogenesis of AD}

25 Puertollano R, Kiselyov K: TRPMLs: in sickness and in health. Am J Physiol Renal Physiol 2009;296:F12451254.

26 Yoo SW, Trout AL, Lovett J, Dickens AM, Xu H, Haughey NJ: A TRPML1 agonist facilitates lysosomal clearance of proteins and lipids in a mouse model of gp120 facilitated amyloid deposition. J Neurovirol 2015;21:S79.

27 Yan S, Yang X, Chen T, Xi Z, Jiang X: The PPAR 7 agonist Troglitazone induces autophagy, apoptosis and necroptosis in bladder cancer cells. Cancer Gene Therapy 2014;21:188-193.

-28 Cerquetti L, Sampaoli C, Amendola D, Bucci B, Masuelli L, Marchese R, Misiti S, De VA, Poggi M, Toscano V: Rosiglitazone induces autophagy in H295R and cell cycle deregulation in SW13 adrenocortical cancer cells. Exp Cell Res 2011;317:1397-1410.

29 Landreth G: PPAR $\gamma$ agonists as new therapeutic agents for the treatment of Alzheimer's disease. Exp Neurol 2006;199:245-248.

30 Guo P, Wang XM, Wang DS: [The research progress of PPAR $\gamma$ in the Neurodegenerative disease]. Sheng li ke xue jin zhan [Progress in physiology] 2015;46:379-382.

31 Kim J, Kundu M, Viollet B, Guan KL: AMPK and mTOR regulate autophagy through direct phosphorylation of Ulk1. Nat Cell Biol 2011;13:132-141.

32 Alers S, Löffler AS, Wesselborg S, Stork B: Role of AMPK-mTOR-Ulk1/2 in the Regulation of Autophagy: Cross Talk, Shortcuts, and Feedbacks. Mol Cell Biol 2012;32:2-11.

-33 Shinojima N, Yokoyama T, Kondo Y, Kondo S: Roles of the Akt/mTOR/p70S6K and ERK1/2 Signaling Pathways in Curcumin-Induced Autophagy. Autophagy 2007;3:635-637.

-34 Yu L, Mcphee CK, Zheng L, Mardones GA, Rong Y, Peng J, Mi N, Zhao Y, Liu Z, Wan F: Termination of autophagy and reformation of lysosomes regulated by mTOR. Nature 2010;465:942-946. 